\title{
Visualizing the Nonhomogeneous Structure of RAD51 Filaments Using Nanofluidic Channels
}

\author{
Louise H. Fornander, ${ }^{\dagger}$ Karolin Frykholm, ${ }^{\ddagger}$ Joachim Fritzsche, ${ }^{\S}$ Joshua Araya, " Philip Nevin," \\ Erik Werner, ${ }^{\perp}$ Ali Çakır, ${ }^{\perp}$ Fredrik Persson, ${ }^{\#}$ Edwige B. Garcin, ${ }^{\triangleright}$ Penny J. Beuning, ${ }^{\|}$Bernhard Mehlig, ${ }^{\perp}$ \\ Mauro Modesti, $\diamond$ and Fredrik Westerlund*, \\ ${ }^{\dagger}$ Department of Chemistry and Chemical Engineering, ${ }^{\ddagger}$ Department of Biology and Biological Engineering, and ${ }^{\S}$ Department of \\ Applied Physics, Chalmers University of Technology, 41296 Gothenburg, Sweden \\ "Department of Chemistry and Chemical Biology, Northeastern University, Boston, Massachusetts 02115, United States, \\ ${ }^{\perp}$ Department of Physics, University of Gothenburg, 41296 Gothenburg, Sweden \\ \# Department for Cell and Molecular Biology, Science for Life Laboratory, Uppsala University, 75124 Uppsala, Sweden

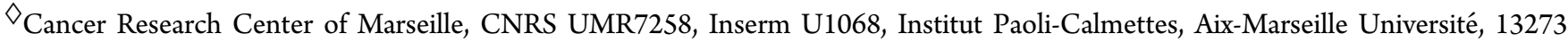 \\ Marseille, France
}

Supporting Information

\begin{abstract}
RAD51 is the key component of the homologous recombination pathway in eukaryotic cells and performs its task by forming filaments on DNA. In this study we investigate the physical properties of RAD51 filaments formed on DNA using nanofluidic channels and fluorescence microscopy. Contrary to the bacterial ortholog RecA, RAD51 forms inhomogeneous filaments on long DNA in vitro, consisting of several protein patches. We demonstrate that a permanent "kink" in the filament is formed where two patches meet if the stretch of naked DNA between the patches is short. The kinks are readily seen in the present microscopy approach but would be hard to identify using conventional single DNA molecule techniques where the DNA is more stretched. We also demonstrate that protein patches separated by longer stretches of bare DNA roll up on each other and this is visualized as transiently overlapping filaments. RAD51 filaments can be formed at several different conditions, varying the cation $\left(\mathrm{Mg}^{2+}\right.$ or $\left.\mathrm{Ca}^{2+}\right)$, the DNA substrate (single-stranded or double-

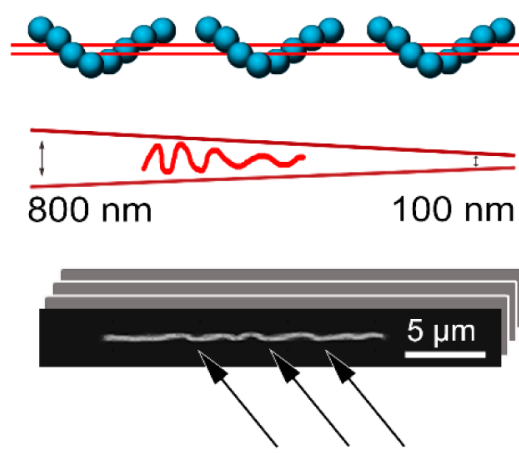
stranded), and the RAD51 concentration during filament nucleation, and we compare the properties of the different filaments formed. The results provide important information regarding the physical properties of RAD51 filaments but also demonstrate that nanofluidic channels are perfectly suited to study protein-DNA complexes.
\end{abstract}

\section{INTRODUCTION}

Single DNA molecule techniques have during the last two decades revolutionized our understanding of how proteins interact with DNA. Using tools such as optical ${ }^{1}$ and magnetic ${ }^{2}$ tweezers, potentially in combination with fluorescence microscopy, ${ }^{3}$ the dynamics of protein-DNA interactions has been characterized in great detail. During the last 10 years, nanofluidic techniques have been used for studying single DNA molecules. ${ }^{4,5}$ Biomacromolecules, such as DNA, can be stretched in narrow channels without tethering the molecule to beads or surfaces, meaning that any long DNA can be studied and enabling studies of processes occurring on the ends of the DNA. Nanofluidics has, for example, been used for DNA polymer-physics studies ${ }^{6}$ and for optical DNA mapping. However, only a limited number of studies on proteins interacting with DNA have been conducted in nanofluidic channels. $^{9-16}$ One of the main reasons for this is the large surface-to-volume ratio in the channels, leading to extensive nonspecific sticking of proteins to the channel walls. This problem was recently solved by passivating the negatively charged silica surface of the nanochannels with a zwitterionic lipid bilayer. ${ }^{17,18}$ Using lipid passivation, the physical properties of the bacterial recombination protein RecA, which forms micrometer-long nucleoprotein filaments around DNA, were studied. $^{15}$ The persistence length of the RecA-DNA filament could be determined by using nanochannels with dimensions corresponding to the Odijk regime, ${ }^{19}$ in which the polymer is confined to so very narrow dimensions that it cannot fold back on itself. At wider dimensions, the polymer enters the extended de Gennes regime. ${ }^{20-22}$ In this regime, the channel is large enough for the polymer to fold back, and its extension is determined by repulsive interactions between different segments. By tailoring the dimensions of the nanochannels it is possible to probe different characteristics of the protein-DNA complex of interest. 
In this paper we compare filaments formed on DNA by human RAD51 proteins with the previously studied bacterial RecA ortholog. ${ }^{15}$ Both RAD51 and RecA catalyze DNA strand exchange, a reaction central in homologous recombination. In this process, the proteins assemble into a right-handed helical filament around single-stranded DNA (ssDNA) (Figure 1A),
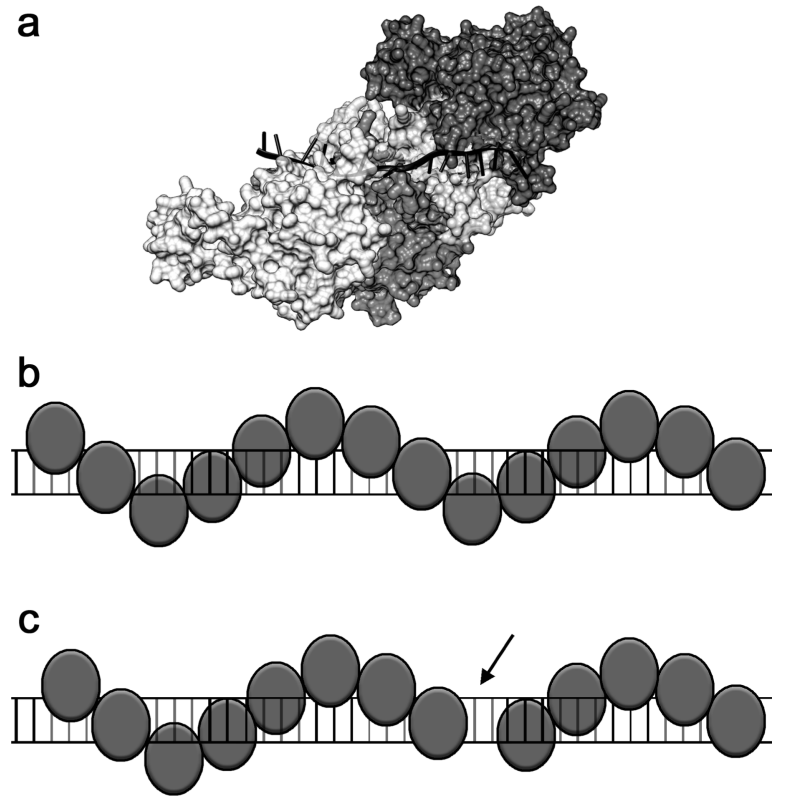

forming the catalytic entity that promotes homology recognition within an intact double-stranded DNA (dsDNA), and subsequently performs the strand exchange reaction. ${ }^{23,24}$ During filament formation, divalent cations, such as $\mathrm{Mg}^{2+}$ or $\mathrm{Ca}^{2+}$, as well as ATP, are needed. When ATP is incorporated into the filament at every monomer-monomer interface, the pitch of the right-handed helical filament is approximately 100 $\AA{ }^{9-16,25-27}$ while the filament is more compressed when ATP is hydrolyzed to $\mathrm{ADP} .^{28,29} \mathrm{ATP}$ hydrolysis triggers RecA dissociation from both ss- and dsDNA ${ }^{30}$ as well as RAD51 dissociation from ssDNA, whereas RAD51 dissociation from dsDNA is comparatively very slow. ${ }^{31-33} \mathrm{Ca}^{2+}$ ions reduce the rate of ATP hydrolysis by RAD51 and thereby stabilize the RAD51-ssDNA filament. ${ }^{34}$ Furthermore, since $\mathrm{Ca}^{2+}$ is a somewhat larger counterion than $\mathrm{Mg}^{2+}$, it aligns the otherwise unstructured bases of $\mathrm{ssDNA}^{35}$ and also increases the torsional stiffness of the filament. ${ }^{36}$ RAD51 and RecA readily form filaments on both ssDNA and dsDNA in vitro. However, in vivo the target for RAD51 filament formation is ssDNA and RAD51-covered dsDNA results from homology recognition and the subsequent strand exchange.

Polymerization of both RecA and RAD51 on DNA involves a nucleation step where 4-6 protein monomers bind to DNA, followed by filament growth. ${ }^{37-40}$ For RecA the intrinsic nucleation is relatively slow compared to the fast and highly cooperative filament growth, ${ }^{41}$ resulting in long continuous
RecA-DNA filaments. For RAD51, by contrast, the balance between nucleation and filament growth is more complex. First, the cooperativity of the RAD51 filament growth is much lower than that of RecA. ${ }^{37,40,41}$ Second, RAD51 filament formation is nucleation limited at low protein concentrations and growth limited at higher concentrations. ${ }^{37}$ Thus, RAD51 moves toward the cooperative behavior demonstrated by RecA at low nucleation concentrations. Due to the low polymerization cooperativity and slow filament disassembly (in the absence of ATP hydrolysis), RAD51 polymerization extends from several nucleation sites, forming small discontinuous protein filaments, or "patches", along the DNA molecule. ${ }^{31,40}$ There is naked DNA of various lengths between these patches, the shortest possible distance being one base pair, but with the two adjacent filaments "out of register" to each other. The term out of register refers to the fact that each RAD51 monomer covers three base pairs ${ }^{17,42,43}$ and there is hence a possibility that two growing filaments that meet may have one or two unbound base pairs between them (see Figure 1B and 1C).

We here investigate the physical properties of RAD51 filaments, formed on both dsDNA and ssDNA, with either $\mathrm{Ca}^{2+}$ or $\mathrm{Mg}^{2+}$ as counterion, when confined to nanofluidic channels. In particular, we characterize filaments with neighboring protein patches that are out of register and relate the physical properties of the formed filaments to the nucleation concentration. We observe that RAD51 forms stationary kinks on the DNA via protein-protein interactions between two adjacent protein patches. We discuss how these kinks depend on the conditions during filament formation and also their potential biological importance.

In addition to revealing novel information about RAD51 filaments on DNA, our study also shows the versatility of nanochannels as tools for studying protein-DNA interactions. We show that nanochannels can be used to detect heterogeneities along a protein-DNA filament, both regarding the actual protein coverage but also in the physical properties of the filament. Several of these features would be very difficult to detect using traditional single DNA molecule techniques in which the DNA is more stretched out.

\section{EXPERIMENTAL SECTION}

Sample Preparation. RAD51 and RecA were labeled with AlexaFluor-555 on C319 and ATTO-488 on the N-terminus, respectively, as described in ref 31 and subsequently stored at $-80{ }^{\circ} \mathrm{C}$. Nucleoprotein filaments were prepared by mixing $\lambda$-DNA (48 502 base pairs (bp), New England Biolabs) or long (>10 000 bases) single-stranded DNA (ssDNA) produced by the rolling circle replication assay described by Gibb et al. ${ }^{44}$ using TempliPhi (GE Life Sciences) with fluorescently labeled proteins to a final concentration of $0.8 \mu \mathrm{M}$ bases or bp DNA and $0.8 \mu \mathrm{M}$ RAD51 or $0.8 \mu \mathrm{M}$ RecA. The buffer used in the nanofluidic experiments was 44.5 $\mathrm{mM}$ Tris-borate and $1 \mathrm{mM}$ EDTA at $\mathrm{pH} 7.5$ (for RAD51) or $\mathrm{pH} 7.0$

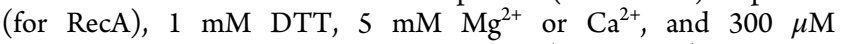
nonhydrolyzable ATP analogues AMP-PNP (for RAD51) or ATP $\gamma$ S (for RecA). The sample solutions also contained $50 \mathrm{mM} \mathrm{KCl}$ from dilution of the protein stock buffer. The samples were incubated for at least $2 \mathrm{~h}$ at room temperature (RAD51) or $2 \mathrm{~h}$ at room temperature followed by overnight incubation at $4{ }^{\circ} \mathrm{C}$ (RecA) before imaging. For experiments in which the effect of RAD51 concentration in the initial nucleation step was examined, the initial protein concentration added to the DNA was decreased to a ratio of 1:600 protein to DNA (bases/ bp). After $1 \mathrm{~h}$ of incubation, the protein concentration was increased in steps until the final protein to DNA (bases/bp) ratio was 1:1. The complete protein addition scheme for these experiments was as follows: initially protein was added to DNA to a ratio of 1:600 
followed by $1 \mathrm{~h}$ incubation, thereafter protein addition to a ratio of 1:60 followed by $1 \mathrm{~h}$ incubation, protein addition to a ratio of $1: 3$ followed by $1 \mathrm{~h}$ incubation, and finally protein addition to the ratio $1: 1$ followed by $2 \mathrm{~h}$ incubation. For all samples the final concentrations were $0.8 \mu \mathrm{M}$ RAD51 and $0.8 \mu \mathrm{M}$ bases/bp DNA. For each experimental condition; at least 20 individual filaments were randomly chosen for imaging and subsequent analysis.

Nanofluidic Setup. The nanofluidic chips were fabricated in fused silica as described elsewhere. ${ }^{4}$ The chip consists of two pairs of microchannels, spanned by funnel-shaped nanochannels with a crosssection of $100-800 \times 140 \mathrm{~nm}^{2}$ (width $\times$ depth) and a length of 500 $\mu \mathrm{m}$ (Figure 2A). The sample is loaded into one of the four reservoirs

a

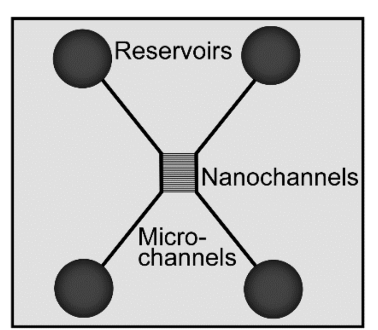

wide end
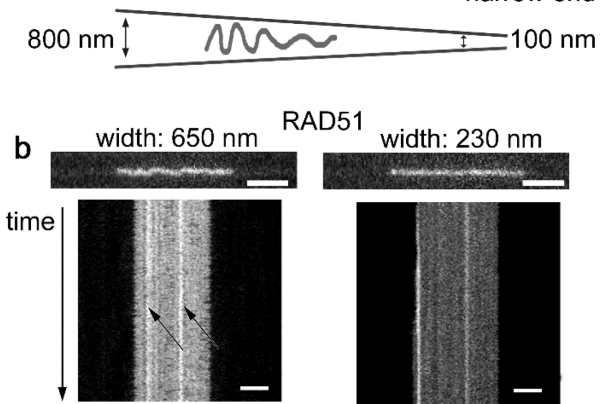

RAD51

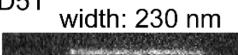

C

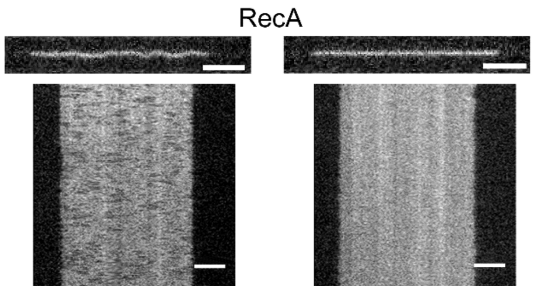

Figure 2. (a) Schematic picture of the nanofluidic chip design and a close up of a nanofunnel. Between the two microchannel systems there is an array of nanochannels, in this study designed as nanofunnels, to which a molecule of interest can be directed by pressure-driven flow. The wide end of the $140 \mathrm{~nm}$ deep funnel is $800 \mathrm{~nm}$ wide, and the narrow end is $100 \mathrm{~nm}$ wide. The length of the funnel is $500 \mu \mathrm{m}$ (not drawn to scale). (b) Snapshot and center-aligned kymographs of a RAD51-coated dsDNA inside the $140 \mathrm{~nm}$ deep nanofunnel at a width of 650 (left) and $230 \mathrm{~nm}$ (right), respectively. The complex was formed in the presence of AMP-PNP and $\mathrm{Mg}^{2+}$ on dsDNA. The RAD51 is in excess compared to dsDNA (protein to bp ratio is 1:1). (c) Same as b but for a RecA-coated dsDNA where ATP $\gamma \mathrm{S}$ was used as nonhydrolyzable ATP analogue. For both the RAD51 and the RecA filaments, a stack of 200 images was taken during a period of 6 s. Scale bars are $5 \mu \mathrm{m}$, and the four corresponding videos are to be found in Supporting Video 1.

that are connected to the microchannels. The protein-DNA filaments are moved inside the chip via the microchannel into the nanochannels by flow generated by applying pressure at the inlets of the microchannel. This allows one to choose a specific position for a filament of interest in the nanochannel and thereby let it reside, nonattached, in a precise confinement. Because of the shallowness of the channel, the filament is mostly moving in $2 \mathrm{D}$ in the focal plane.
Also, since the channel is much longer than the filament, the difference in confinement throughout the length of one protein-DNA complex is negligible.

To avoid nonspecific binding of the protein to the negatively charged silica surface, the chip was coated with a lipid bilayer consisting of 99\% 1-palmitoyl-2-oleoyl-sn-glycero-3-phosphocholine (POPC, Avanti) and 1\% N-(fluorescein-5-thiocarbamoyl)-1,2-dihexadecanoyl-sn-glycero-3-phosphoethanolamine, triethylammonium salt (fluorescein-DHPE, Invitrogen) (for RAD51) or $1 \%$ lissamine rhodamine B 1,2-dihexadecanoyl-sn-glycero-3-phosphoethanolamine, triethylammonium salt (rhodamine-DHPE, Invitrogen) (for RecA), before samples were introduced. The coating procedure is described in detail elsewhere. ${ }^{18}$

The filaments were imaged using an epifluorescence microscope (Zeiss AxioObserver.Z1) equipped with a Photometrics Evolve EMCCD camera and a 100X oil immersion TIRF objective (NA = 1.46) from Zeiss. Using the software AxioVision, 200 subsequent images were recorded with an exposure time of $20 \mathrm{~ms}$ and an interval between the images of $30 \mathrm{~ms}$.

During imaging, the pressure of the system was turned off in order to let the protein-DNA complex reside without any fluid flow in the nanochannels. However, occasionally a slight movement of the complex within the channel was observed, probably due to a small pressure offset in the system, but this was accounted for during the analysis.

Data Analysis. Snapshots from videos were extracted in ImageJ (www.imagej.com). Kymographs were constructed from these images using a custom-written MATLAB program. Heat maps were created with the help of the open source image processing algorithm suite scikit-image for Python. ${ }^{45}$ This image processing procedure consists of three main steps: denoising, thinning to a 1 pixel thick skeleton, and cleaning the resulting skeleton. In the first step, the images were denoised by a total-variation denoising algorithm. ${ }^{46}$ The denoised images were subsequently converted to binary images by comparing the pixel value to a threshold level. The binary images were passed through a median filter in order to remove corners that complicate the thinning. The images were thinned down to a 1 pixel thick path using the skeletonization algorithm provided in the scikit-image library. Finally, spurious artifacts such as loops, branches, and free dots were identified and resolved using custom algorithms. The resulting path is identified as the instantaneous configuration of the molecule. The free parameters of the algorithm were empirically adjusted to ensure both that the configurations yield a visually satisfying description of the original video frame and that the contour length of the path changes little from one frame to the next. Finally, the instantaneous configurations from the different frames were superimposed, yielding the heat maps plotted in Figures 3C, 4, and 6.

\section{RESULTS}

Figure 2 shows microscopy images and corresponding kymographs of human RAD51 filaments compared with images and kymographs of filaments formed by its bacterial ortholog RecA, which is known to form uniform filaments. ${ }^{15}$ Using a funnel-shaped nanochannel, ${ }^{47}$ with a depth small enough for the molecule of interest to stay in focus and to move mainly in two dimensions in the focal plane, we monitored an individual filament at various confinements, allowing us to observe different characteristics of the filaments. In the wide part of the funnel ( $650 \mathrm{~nm}$ wide and $140 \mathrm{~nm}$ deep) the filament was found to undulate between the channel walls, while in the narrow part (230 nm wide and $140 \mathrm{~nm}$ deep) it is stretched out (see Supporting Video 1). In the narrow confinement we detected and characterized intensity variations along the protein-DNA complex, reflecting the protein density distribution along the DNA.

Interestingly, filaments of RAD51 and RecA formed on dsDNA in the presence of $\mathrm{Mg}^{2+}$ and a nonhydrolyzable ATP 
analogue (AMP-PNP for RAD51 and ATP $\gamma \mathrm{S}$ for RecA) behaved differently from each other, both in the wide and in the narrow end of the funnel (Figure 2B and 2C). Near the wide end, both complexes undulated between the walls, but the RAD51 filament had a shorter "wavelength" than RecA (see Supporting Video 2 for more examples). In Odijk's theory for strongly confined polymers, this wavelength is generally referred to as the length of a deflection segment. ${ }^{19}$ Near the narrow end of the funnel, differences in homogeneity and continuity between the two complexes were observed (see Figure 2B and 2C and Supporting Video 1). How the uneven protein coverage is related to the physical properties of the RAD51-DNA complex is discussed in detail below.

Near the wide end of the funnel, we observed two main characteristics in the complexes formed with RAD51 that are not seen for the RecA complexes; "stationary kinks" and "transient overlaps", both related to the formation of RAD51 patches along the DNA. A stationary kink is a permanent bend in the filament that is not part of the filament fluctuations in the transversal direction in the channel. A transient overlap is a region in the filament with high protein intensity that forms and disappears throughout the imaging time. Below we discuss these two features in detail and relate their properties to the presence of protein patches on the DNA.

We will first demonstrate these two phenomena per se, and then describe how our findings depend on the type of counterion present $\left(\mathrm{Mg}^{2+}\right.$ or $\left.\mathrm{Ca}^{2+}\right)$, the nature of the DNA substrate (ssDNA or dsDNA), and the protein concentration during nucleation. To minimize any effects of depolymerization due to ATP hydrolysis, ${ }^{48,49}$ all results presented below were obtained with filaments formed with the nonhydrolyzable ATP analogues AMP-PNP (for RAD51) or ATP $\gamma$ S (for RecA). We confirmed that similar results are obtained for RAD51 filaments on dsDNA in the presence of ATP and $\mathrm{Ca}^{2+}$ (Supporting Video 3), a combination that decreases the rate of ATP hydrolysis and subsequent filament depolymerization. ${ }^{34}$

RAD51 Filaments Form Stationary Kinks on DNA. As mentioned above, RAD51-DNA complexes form stationary kinks. This feature is not observed for RecA (compare the RecA and the RAD51 filaments confined to channels that are $650 \mathrm{~nm}$ wide and $140 \mathrm{~nm}$ deep in Supporting Video 2). There are several ways to visualize such kinks. Figure 3A shows kymographs, where the local protein concentration is directly proportional to the emission intensity, for three individual horizontal pixel rows along the channel (from now on referred to as "line-by-line" kymographs). For a filament that undulates smoothly between the channel walls, such as the RecA filament, all line-by-line kymographs are similar. For the RAD51 complexes, by contrast, there are distinct intensity patterns in these kymographs, predominantly close to the walls of the channel (Figure 3A). An area with high emission intensity in the upper region of the channel corresponds to a low-intensity area in a lower line kymograph at the same vertical position and vice versa, demonstrating the presence of a kink. These kinks persist for $6 \mathrm{~s}$ or more, indicating that they are very stable. The stationary kinks can also clearly be visualized in heat maps constructed from superimposed trajectories of the filaments (Figure 3C, see Experimental Section for details). For a filament that undulates evenly over the width of the channel, as is the case for RecA, the heat map is uniform with no stationary regions in the filament (Figure 3C, right). For RAD51 filaments, on the other hand, the kinks are clearly visible in the heat maps (arrows indicate examples of kinks in Figure 3C, left).

To investigate how stable the kinks on the RAD51-DNA complexes are we moved kink-containing filaments from the wide end of the funnel to the narrow end and back again. In the narrow part, where the confinement is stronger, the filaments are stretched out and, interestingly, the stronger confinement caused some of the kinks to be mechanically removed while others changed position along the filament (Figure 4).

RAD51 Filaments Form Transient Overlaps at Moderate Confinement. Figure 5 shows two RAD51 complexes, one formed on ssDNA and the other one formed on dsDNA, in the wide (650 nm wide) and narrow (230 nm wide) end of the $140 \mathrm{~nm}$ deep nanofunnel. In the wide end (top row Figure 5A and $5 \mathrm{~B}$ ), the filaments have a tendency to form transient regions of higher emission intensity, suggesting that the filaments "roll up" onto themselves. When such a filament is moved to the narrow end of the nanofunnel it is evident that the RAD51 covers the DNA in patches (bottom row Figure 5A and $5 B$ ) and that the roll ups observed in the wide end of the channel appear close to regions where there is no protein bound. Note that there are also areas with higher protein density that do not disappear even though the filaments are strongly confined.

To characterize the high-intensity regions in more detail we constructed intensity histograms based on all pixels in the kymographs (right column Figure 5A and 5B). The background is set to zero, and the main intensity peak is normalized to " 1 ". In these histograms, in addition to the large peak at 1 , we observe a shoulder centered around 1.5-2 in the histograms for the wide end of the funnel (arrows in histograms, top row, Figure $5 \mathrm{~A}$ and $5 \mathrm{~B}$ ), which corresponds to the high-intensity areas in the kymographs. These shoulders are significantly reduced in the histograms for the narrow end of the funnel (bottom row, histograms Figure 5A and 5B), and we conclude that they originate from the transient overlaps. The fact that the intensity rarely increases much above 2 suggests that it is not a completely protein-coated region that creates a "Z-bend" (since this would lead to an intensity value on the order of 3) but rather that two protein patches, connected by naked DNA, are positioned on top of each other (cartoons, Figure 5C). We also see no evidence that the appearance and disappearance of overlaps at different positions along the DNA are correlated, they are rather individual, local phenomena that do not depend on whether another overlap is formed or disappears at the same time.

RAD51 Nucleation Concentration, Counterions, and DNA Substrate Affect the Physical Properties of the Filament. As a next step we investigated how the physical properties of the RAD51-DNA complexes, and in particular the presence of kinks, are affected by the DNA substrate (ss or ds), the counterion present $\left(\mathrm{Mg}^{2+}\right.$ or $\left.\mathrm{Ca}^{2}\right)$, and the protein concentration at the initial nucleation step. A lower concentration of RAD51 during the first stage of the filament assembly reaction, the nucleation, is expected to result in fewer nucleation sites along the DNA and thus promote formation of longer RAD51 patches and, if our model is correct, fewer kinks.

In the results presented so far, filaments were formed by mixing RAD51 and DNA at a protein to bases/bp ratio of 1:1; hence, there is an excess of RAD51 protein in the solution since the binding stoichiometry of RAD51 is approximately one protein monomer per $3-4$ bases/bp. ${ }^{17,42,43}$ To reduce the number of nucleation sites, RAD51 and DNA were instead 
a

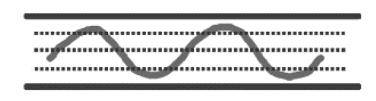

RAD51
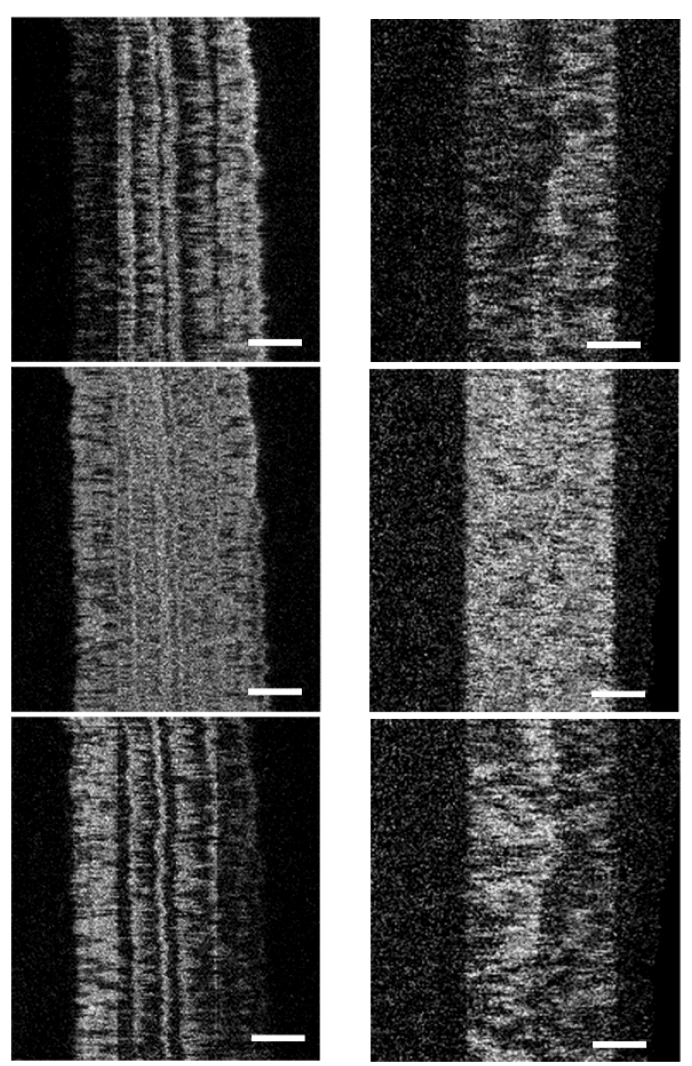

b
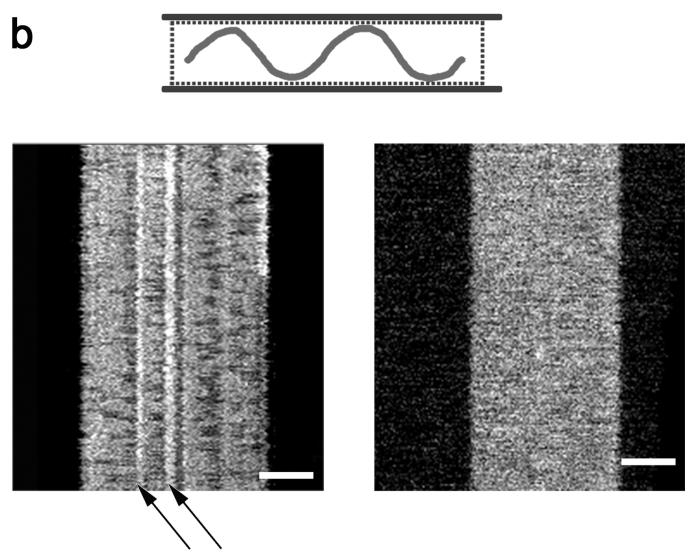

C
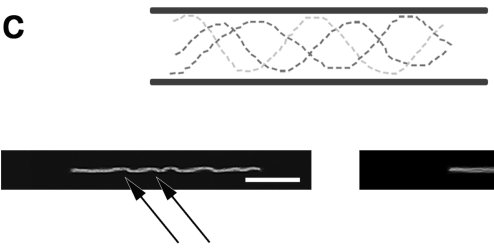

Figure 3. (a) Three "line-by-line" kymographs for RAD51 (left column) and RecA (right column) filaments formed on dsDNA in the presence of $\mathrm{Mg}^{2+}$ and AMP-PNP (RAD51) or ATP $\gamma \mathrm{S}$ (RecA), respectively, generated by creating a kymograph for individual horizontal pixel rows along the channel (three dotted lines in schematic figure). Corresponding average kymographs for the filaments in a are shown in b. (c) Heat maps from 200 images
Figure 3. continued

(taken over a time period of $6 \mathrm{~s}$ ) of the RAD51- and RecA-dsDNA complexes. For comparison, the same complexes are depicted in a-c. The RAD51 complex was confined to channels of $650 \mathrm{~nm}$ width and $140 \mathrm{~nm}$ depth and the RecA filament to channels of $710 \mathrm{~nm}$ width and $140 \mathrm{~nm}$ depth, and scale bars are $5 \mu \mathrm{m}$. Corresponding videos are shown in Supporting Video 4.

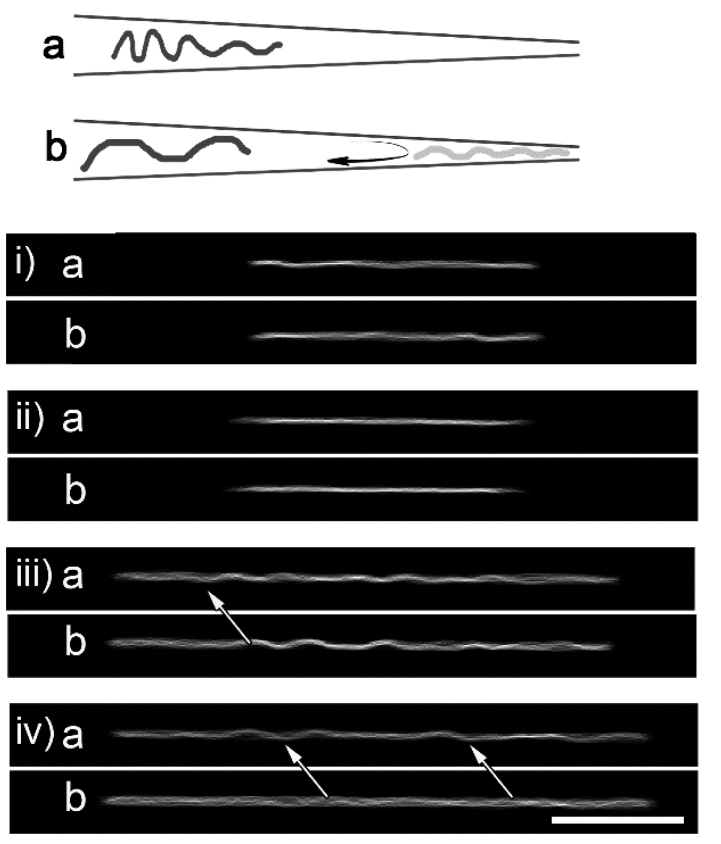

Figure 4. Stationary kinks may be mechanically moved or removed by moving the RAD51 filaments from the wide end of the nanofunnel to the narrow end. Cartoons above the heat maps of four individual filaments (i-iv) demonstrate the "treatment": if the filaments reside in the wide $(650 \mathrm{~nm})$ end of the $140 \mathrm{~nm}$ deep funnel (a) or if the filaments are observed at the same confinement but have previously been experiencing the confinement at the narrow end of the funnel $(230 \mathrm{~nm})(\mathrm{b})$. Heat maps of the four different RAD51 complexes in the wide end of the nanofunnel show that filaments iii and iv have clear kinks (a) and that most of these kinks are gone, or moved, after being in the narrow part of the channel (b). The stack of images consists of 200 frames with a total imaging time of $6 \mathrm{~s}$, and the scale bar is $5 \mu \mathrm{m}$. All complexes were assembled on dsDNA in the presence of $\mathrm{Mg}^{2+}$ and AMP-PNP. Corresponding videos are to be found in Supporting Video 5.

mixed and incubated at an initial ratio of 1:600 (protein:bases/ $\mathrm{bp}$ ), and thereafter the concentration of RAD51 was increased in steps until the sample had a final protein to DNA ratio of 1:1 (for detailed procedure see Experimental Section). The two different nucleation conditions (initial ratios 1:1 and 1:600) were both investigated with different counterions $\left(\mathrm{Mg}^{2+}\right.$ or $\mathrm{Ca}^{2+}$ ) and DNA substrates (ss or ds).

When $\mathrm{Mg}^{2+}$ was used as counterion, there was a significant effect when the nucleation concentration of RAD51 was decreased (Figure 6A). On dsDNA, the stationary kinks observed for complexes formed at a protein:bp ratio of $1: 1$ (see Figures 3 and 4) almost disappear completely when the initial RAD51 concentration was decreased to a protein:bp ratio of 1:600 (Figure 6A, left column). Since lowering the initial protein concentration should lead to fewer nucleation sites and fewer but longer patches, the appearance of the filaments agrees well with the interpretation that the stationary kinks are formed 


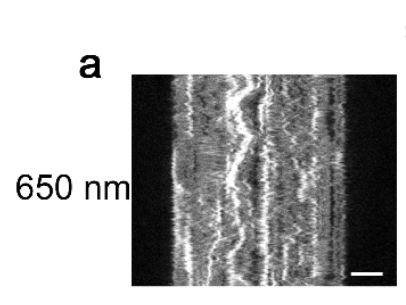

SsDNA
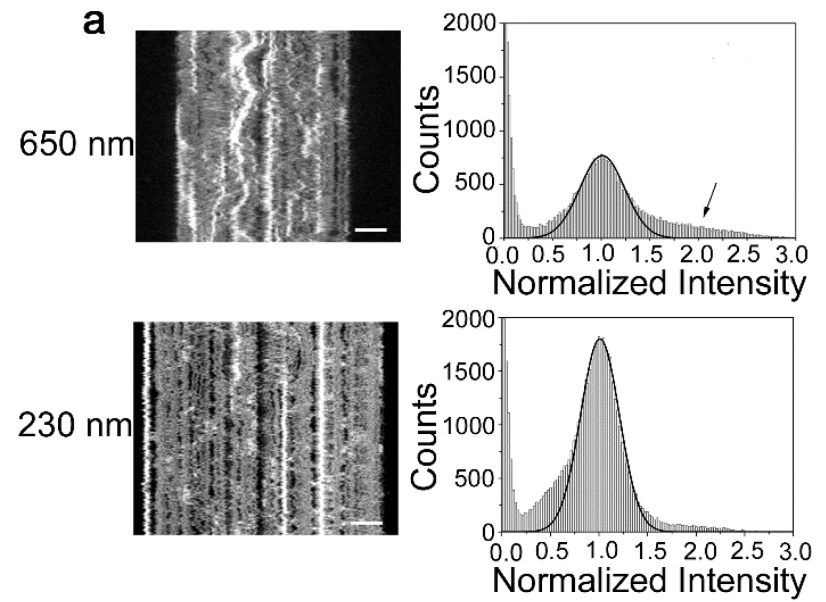

b

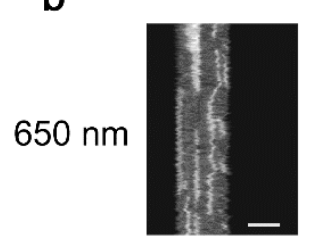

dsDNA
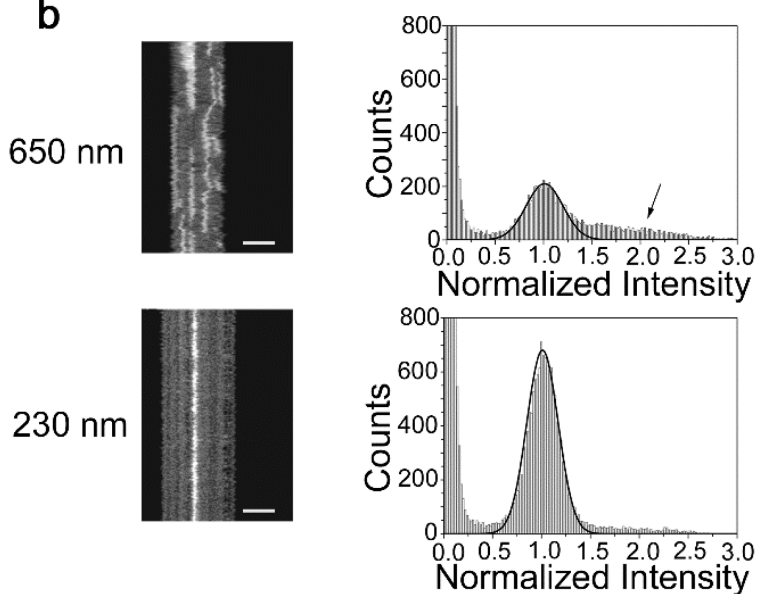

C

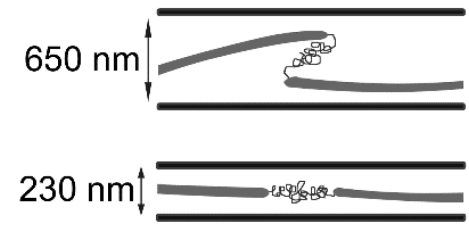

Figure 5. Kymographs aligned by their center of mass and stretched to equal length in each row (left) and their corresponding emission intensity histograms (right) for two RAD51 filaments. Filaments were formed in the presence of AMP-PNP and $\mathrm{Mg}^{+}$on ssDNA (a) and dsDNA (b), respectively, and observed at two different widths of the $140 \mathrm{~nm}$ deep nanofunnel, 650 and $230 \mathrm{~nm}$, respectively. At $650 \mathrm{~nm}$ width, the filaments have a tendency to roll up and form transient overlaps, giving the high-intensity areas that disappear and reappear in the kymographs. When the filaments are stretched out in the narrower confinement (230 nm width), a heterogeneity in protein coverage (fluorescence intensity) becomes evident, demonstrating that the RAD51 protein has formed fluorescent patches along the DNA (bright areas in the kymographs) with gaps in between (dark areas in the kymographs). In the histograms for filaments in channels of $650 \mathrm{~nm}$ width and $140 \mathrm{~nm}$ depth, there are shoulders at higher intensities (see arrows), which are missing in the histograms for filaments at the narrow confinement $(230 \mathrm{~nm}$ width and $140 \mathrm{~nm}$ depth). Schematic Gaussians centered at 1.0 are shown to guide the eye. (c) Cartoons that demonstrate schematically the suggested organization of the RAD51 patches in the wide confinement and the narrow confinement. Kymographs consist of 200 frames with a total imaging time of $6 \mathrm{~s}$, and scale bars are $5 \mu \mathrm{m}$. Four corresponding videos can be found in Supporting Video 6.
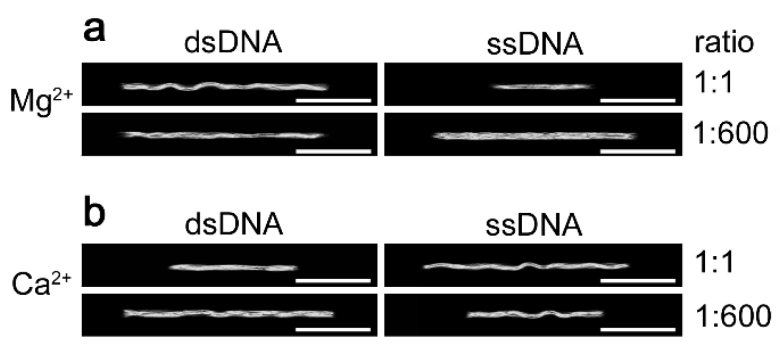

Figure 6. Representative heat maps demonstrating the effect the initial concentration of RAD51 has on the presence of stationary kinks in filaments formed on ssDNA and dsDNA with $\mathrm{Mg}^{2+}$ (a) or $\mathrm{Ca}^{2+}$ (b) present. Ratios indicated to the right are the number of RAD51 monomers to the number of bases/bp in the initial nucleation step during the assembly reaction. Final concentrations and ratios are the same (0.8 $\mu \mathrm{M}$ RAD51 and $0.8 \mu \mathrm{M}$ bases/bp DNA, protein to DNA ratio 1:1, see the Experimental Section for details) for all filaments. The stack of images consists of 200 frames with a total imaging time of $6 \mathrm{~s}$, and scale bars are $5 \mu \mathrm{m}$. The corresponding video to each heat map is found in Supporting Video 7, and more examples of filaments formed under these conditions are found in Figure S1.

between two adjacent RAD51 patches. Comparing filaments formed on ssDNA and dsDNA shows that the complexes formed on ssDNA display fewer kinks, and hence, if our argument is true, lesser and longer patches have been formed. When decreasing the initial concentration of RAD51 to 1:600 on ssDNA in order to reduce the number of nucleation sites, the filaments display even fewer stationary kinks (Figure 6A).

Interestingly, the effect of the nucleation concentration when $\mathrm{Ca}^{+}$was present was not at all similar to that seen in the presence of $\mathrm{Mg}^{2+}$. When lowering the nucleation ratio from 1:1 to 1:600 with $\mathrm{Ca}^{2+}$ present, more stationary kinks appeared for dsDNA (Figure 6B, left column). Also, in the presence of $\mathrm{Ca}^{2+}$, more kinks seemed to occur in filaments formed on ssDNA compared to dsDNA (Figure 6B). Thus, in both of these regards, decreasing the RAD51 nucleation ratio and changing the DNA substrate from ds to ss, $\mathrm{Ca}^{2+}$ has the opposite effect on the physical properties of the filament compared to $\mathrm{Mg}^{2+}$.

In Table 1 we summarize our observations on how the presence of kinks correlates to the different conditions investigated (i.e., dsDNA/ssDNA, $\mathrm{Mg}^{2+} / \mathrm{Ca}^{2+}$, and 1:1/1:600 nucleation ratio). We also include a qualitative assessment of how common the presence of transient overlaps is. We note

Table 1. Dominant Feature Detected in RAD51 Filaments Formed on ssDNA or dsDNA in the Presence of $\mathrm{Ca}^{2+}$ or $\mathrm{Mg}^{2+}$ at Different Nucleation Concentrations of RAD51 (1:1 or 1:600 RAD51 monomers to bases $/ \mathrm{bp})^{a}$

$\begin{array}{ccccc}\text { counterion } & \text { DNA substrate } & \text { nucleation ratio } & \text { kinks } & \text { overlaps } \\ \mathrm{Mg}^{2+} & \text { dsDNA } & 1: 1 & ++ & - \\ & & 1: 600 & - & + \\ & \text { ssDNA } & 1: 1 & - & ++ \\ & & 1: 600 & + & ++ \\ \mathrm{Ca}^{2+} & \text { dsDNA } & 1: 1 & - & ++ \\ & & 1: 600 & + & - \\ & \text { ssDNA } & 1: 1 & + & + \\ & & 1: 600 & + & +\end{array}$

$a_{+}$and - indicate if filaments formed at each condition do or do not exhibit the specific feature, respectively, and ++ indicates if the feature is common. A minimum of 20 filaments has been observed for each condition. 
that an individual filament rarely possesses both kinks and transient overlaps simultaneously; instead, there is usually one feature that dominates in each complex for each experimental condition. However, different filaments in the same sample can contain either kinks or overlaps.

Persistence Length Analysis. In a previous study we used nanofunnels to determine the persistence length of RecA filaments. ${ }^{15}$ The analysis was based on Odijk theory for polymers confined to channels with dimensions smaller than their persistence length. ${ }^{19}$ In this regime the extension of a polymer depends on the channel dimensions as follows

$$
r=L\left(1-B\left[\left(\frac{D_{1}}{P}\right)^{2 / 3}+\left(\frac{D_{2}}{P}\right)^{2 / 3}\right]\right)
$$

Here $r$ is the extension of the filament in the channel, $L$ is the contour length, $P$ is the persistence length, $D_{1}$ and $D_{2}$ are the dimensions of the channel cross-section, and the constant $B$ is numerically estimated to $0.09137 .{ }^{50}$ The theory assumes that the object under study is homogeneous, complicating the analysis of RAD51 filaments. According to the observations above (Table 1), filaments formed on dsDNA in the presence of $\mathrm{Mg}^{2+}$ at a nucleation ratio of $1: 1$ are fairly homogeneous and, importantly contain few regions of naked DNA that would be much more flexible than the actual nucleoprotein filament. In Figure 7 we have fitted the extensions of filaments formed at

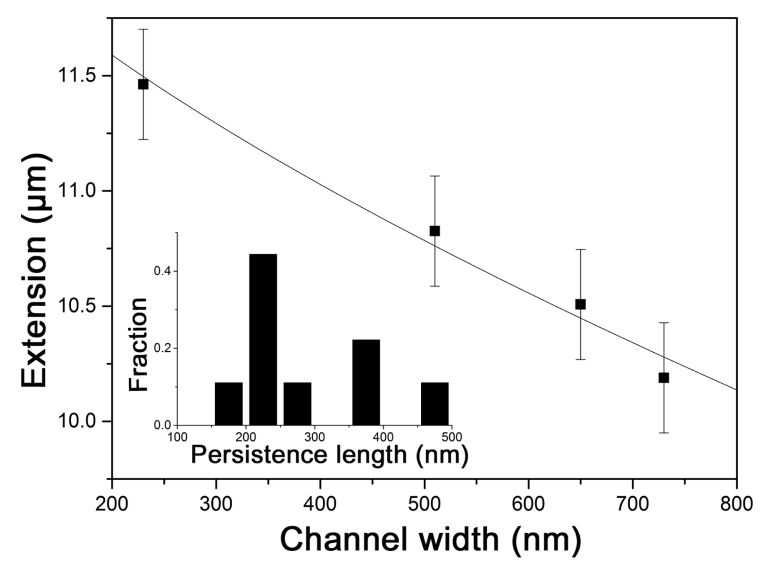

Figure 7. Extension of a RAD51-dsDNA filament formed in the presence of $\mathrm{Mg}^{2+}$ and at an initial protein to DNA ratio of 1:1 versus channel width in a $140 \mathrm{~nm}$ deep nanofunnel (solid squares). The solid line is the fit to eq 1 , and this specific filament yields a persistence length of $297 \mathrm{~nm}$. (Inset) Persistence lengths determined for nine RAD51 filaments.

these conditions, for different degrees of confinement, to eq 1 by a least-squares method with two unknown parameters, $L$ and $P$ (see also Supporting Information). We find that the average persistence length of the RAD51 filaments (formed in the presence of AMP-PNP and $\mathrm{Mg}^{2+}$ on dsDNA) is $296 \pm 89 \mathrm{~nm}$. If we only consider filaments that fall under the main peak of the histogram (inset of Figure 7), we instead obtain a persistence length of $239 \pm 27 \mathrm{~nm}$, in the same range as previously reported values. ${ }^{27,37,40}$

\section{DISCUSSION}

It is well established that, due to a competition between nucleation and polymerization, RAD51 filaments are discontinuous and consist of patches along both ssDNA and
dsDNA. ${ }^{31,37,40}$ In this study we investigated how such patches affect the physical properties of the filament and in particular what happens when two patches meet. We did this by stretching the filaments in nanofluidic channels and visualizing them using fluorescence microscopy.

We previously used funnel-shaped nanochannels to determine the persistence length of the nucleoprotein filament of the prokaryotic RAD51 ortholog RecA. However, it is not straightforward to determine the persistence length of the RAD51 filaments with the method used earlier, because RAD51 does not coat the DNA homogeneously. Instead, it forms several shorter nucleoprotein filaments ("patches") on the DNA with stretches of naked DNA in between ${ }^{31,33,40}$ that are much more flexible than the actual nucleoprotein filament. However, for the condition of an initial protein to DNA ratio of $1: 1$, dsDNA and $\mathrm{Mg}^{2+}$, we were able to conduct the same persistence length analysis as in our previous study, ${ }^{15}$ since these filament do not demonstrate long stretches of naked DNA. We obtained a persistence length of $239 \pm 27 \mathrm{~nm}$, consistent with a previous report of a persistence length of 190 $\pm 60 \mathrm{~nm}$ in the case of dsDNA with AMP-PNP and $\mathrm{Mg}^{2+37}$ as well as other reports of persistence lengths ranging from $268 \pm$ 17 to $460 \pm 40 \mathrm{~nm}$ depending on the nucleotide cofactor, DNA, and cation used. ${ }^{37,40}$

In the wide end of the funnel we detected stationary kinks in the RAD51-DNA complexes. We argue that these are present because two adjacent RAD51 patches can make strong protein-protein interactions when there is a short stretch of naked DNA between them. We detected the kinks in the heterogeneously covered RAD51 filaments, but they are not present in the homogeneously covered RecA filaments, in agreement with previous AFM observations. ${ }^{31}$ Probably, when one polymerizing RAD51 filament reaches an adjacent one, potentially growing in the opposite direction, the two filaments are not always able to merge, either because there are 1 or 2 bases/bp between them or the two adjacent RAD51 monomers are not in the right pitch relative to each other to form a long continuous filament.

Interestingly, a significant fraction of the kinks was mechanically removed by moving the filaments to a more narrow confinement in the nanofunnels. We also observed that for some filaments the kinks moved to another region of the filament. The movement of the kinks along the filament could be explained as strain in the filament that when it is released at one location is causing a kink at another, potentially involving rearrangement of the filament patches along the DNA. Similarly, it has been speculated that a biological function of the regions of naked DNA in the discontinuous RAD51 filaments could be to release topological constraints during the strand exchange process. ${ }^{40}$

We found that the RAD51 filaments that contain longer naked DNA gaps between the protein patches demonstrate a tendency to form transient overlaps in the wide end of the nanofunnel. That it is in fact long naked DNA regions was confirmed by moving the filaments to the narrow end of the funnel, revealing nonfluorescent regions along the DNA corresponding to at least several hundred bases/bp of naked DNA (see Figure 5). We observed that the overlaps possess approximately double the emission intensity and hence double the protein concentration compared to the stretched out single filament and could therefore conclude that two patches lay on top of each other as schematically depicted in Figure 5C and not in a Z-like overlap. Due to the small persistence length of 
ssDNA $\left(P \approx 2.5 \mathrm{~nm}^{51}\right)$, transient overlaps are expected to appear more frequently on that substrate than on $\operatorname{dsDNA}(P \approx$ $50 \mathrm{~nm}^{6}$ ), supporting our hypothesis about their origin.

It has previously been shown that the length of the nucleoprotein filaments formed by RAD51 on DNA depends on a delicate balance between the rate of nucleation and the rate of polymerization. ${ }^{37,40}$ RAD51 has a fast nucleation rate relative to its filament growth ${ }^{31,32,40,49}$ as opposed to RecA that exhibits a much more cooperative filament growth, ${ }^{41}$ and nucleates at multiple sites along the DNA. By lowering the RAD51 concentration fewer nucleation sites can form, ${ }^{37,38}$ in agreement with our observation that more homogeneous filaments are formed at a decreased initial protein concentration. Fewer kinks were detected when the initial concentration was decreased for RAD51-dsDNA filaments in the presence of $\mathrm{Mg}^{2+}$, and the filaments actually behaved very similarly to the continuous complexes formed with RecA. However, the coverage on the dsDNA does not appear to be complete in filaments formed at an initial concentration of 1:600, since some transient overlaps also appear. Probably, the RAD51 patches are longer, due to a decreased number of nucleation sites, but incomplete polymerization results in longer naked DNA regions between the protein patches as well. Also, a more homogeneous filament is detected when ssDNA is used instead of dsDNA in the presence of $\mathrm{Mg}^{2+}$.

The nucleation-polymerization balance seems to depend also on whether $\mathrm{Mg}^{2+}$ or $\mathrm{Ca}^{2+}$ is present. Surprisingly, the results for filaments formed in the presence of $\mathrm{Ca}^{2+}$ were opposite to those obtained with $\mathrm{Mg}^{2+}$ in both regards; no decrease in the number of kinks was detected when the initial concentration was lowered or when dsDNA was exchanged for ssDNA (Figure 6). We observed, in general, that the transient overlaps, stemming from long stretches of bare DNA, are less abundant in the presence of $\mathrm{Ca}^{2+}$, indicating that the nucleoprotein patches are longer. $\mathrm{Ca}^{2+}$ is known to inhibit the ATP hydrolysis activity of RAD51, thereby stabilizing the active form of the nucleoprotein filament and reducing filament disassembly. ${ }^{34}$ In the presence of ATP, alternating the cation is thus a way to regulate the rate of ATP hydrolysis. Mine et al. observed, using magnetic tweezers, that the rate of filament formation on ssDNA in the presence of $\mathrm{Ca}^{2+}$ is different from that in the presence of $\mathrm{Mg}^{2+}$, but they could not discriminate between nucleation and polymerization during the filament formation process. $^{37}$ In a scanning force microscopy study, Ristic et al. observed regular nucleoproteins formed under suppressed ATP-hydrolysis conditions (ATP/ $\mathrm{Ca}^{2+}$ and AMP$\left.\mathrm{PNP} / \mathrm{Mg}^{2+}\right),{ }^{52}$ and van der Heijden et al. reported on elongated DNA with a high degree of RAD51 coverage in the presence of ATP and $\mathrm{Ca}^{2+}$ from magnetic tweezers experiments and Monte Carlo simulations. ${ }^{40}$ Under the experimental conditions used in the present study, ATP hydrolysis is eliminated by the use of the ATP analogue AMP-PNP, and the differences we observed between nucleoprotein filaments formed in the presence of $\mathrm{Mg}^{2+}$ and $\mathrm{Ca}^{2+}$ are due to effects of the cation itself. According to our findings, the critical initial RAD51 concentration that decreases the number of protein patches along the DNA strand is not reached at an initial protein to bases/bp ratio of 1:600 in the presence of $\mathrm{Ca}^{2+}$, but it is with $\mathrm{Mg}^{2+}$. It is thus evident that the balance between the rate of nucleation and polymerization is different between the two counterions, causing the number of RAD51 patches and subsequently the number of kinks and transient overlaps to be different as well.
The presence of $\mathrm{Mg}^{2+}$ or $\mathrm{Ca}^{2+}$, ssDNA or dsDNA, as well as different initial concentrations of RAD51 alters the length and the number of fluorescent patches along the DNA. They also change the length of the uncovered, or naked, DNA between the protein patches. We speculate that the presence of uncoated DNA of various lengths between protein-covered patches is the origin of both of the two phenomena detected, kinks and overlaps. Shorter stretches of naked DNA enable adjacent protein patches to interact, resulting in filaments with stationary kinks, while longer stretches of naked DNA permit the protein patches to fold back on each other in the wide part of the funnel, resulting in transient overlaps (Figure 5 and Supporting Figure S2). Interestingly, individual filaments exhibit either stationary kinks or transient overlaps, but filaments displaying either of the two features can be detected in a single sample prepared at a certain condition.

It remains to be demonstrated if the kinks have biological relevance. Several accessory proteins are required to help RAD51 perform its function in vivo, ${ }^{53-55}$ and these could regulate the assembly (and the subsequent disassembly) of the catalytically competent RAD51 nucleoprotein filaments by controlling the nucleation and/or filament elongation steps. It is therefore plausible that there is a specific machinery in cells that makes sure that the kinks are not formed. On the other hand, the fact that kinks are formed on RAD51 filaments in vitro is important even if they are not present in vivo. This is because many strand exchange experiments are performed in vitro on simple samples where proteins that possibly remove kinks are not present. Another possibility is that the kinks are important in vivo, for example, to provide flexibility or a means to store or to release topological strain in the nucleoprotein filaments, thereby facilitating homology search and/or strand exchange. Whether this is the case or not remains to be investigated.

In a broader context, our study demonstrates the usefulness of nanofluidic channels for investigating single protein-DNA complexes in solution. We observe heterogeneities in RAD51 coverage along the DNA that correspond to physical properties of the filament, properties that are relevant for many different DNA-binding proteins. The fact that we do not need to tether the DNA to beads or surfaces means that we can study protein-DNA complexes and potentially other biopolymers at low forces that are not readily accessible with other traditional single DNA molecule techniques. Finally, since no end manipulation of the DNA is required we can potentially use DNA of any origin, even specific DNA or protein-DNA complexes extracted directly from cells.

\section{CONCLUSION}

We characterized the physical properties of RAD51 filaments in solution using funnel-shaped nanofluidic channels. We detected stationary kinks and transient overlaps in the filaments, as a result of the heterogeneous coverage on DNA by RAD51. Our data indicates that the length of the naked DNA between the RAD51 patches determines whether the filament displays kinks or overlaps. No, or very short, naked DNA stretches result in stationary kinks, while longer bare DNA gives rise to transient overlaps. The DNA substrate (ssDNA or dsDNA), the type of cation present $\left(\mathrm{Mg}^{2+}\right.$ or $\left.\mathrm{Ca}^{2+}\right)$, and the initial RAD51 concentration together determine the number of RAD51 patches on the DNA and the length of the naked DNA between them. 


\section{ASSOCIATED CONTENT}

S Supporting Information

The Supporting Information is available free of charge on the ACS Publications website at DOI: 10.1021/acs.langmuir.6b01877.

Additional examples of the superimposed trajectories presented in Figure 6; raw kymographs corresponding to Figure 5 (PDF)

Videos corresponding to the images in Figure 2 (AVI)

Videos corresponding to the images in Figures 2-6 (AVI)

Videos corresponding to the images in Figures 2-6 (AVI)

Videos corresponding to the images in Figure 3 (AVI) Videos corresponding to the images in Figure 4 (AVI) Videos corresponding to the images in Figure 5 (AVI) Videos corresponding to the images in Figure 6 (AVI)

\section{AUTHOR INFORMATION}

\section{Corresponding Author}

*E-mail: fredrik.westerlund@chalmers.se.

\section{Author Contributions}

The manuscript was written through contributions of all authors. All authors have given approval to the final version of the manuscript.

\section{Notes}

The authors declare no competing financial interest.

\section{ACKNOWLEDGMENTS}

This project was supported by the Swedish Research Council Grant [2011-4324 to F.W. and 2013-3992 to B.M.], the Chalmers Area of Advance in Nanoscience and Nanotechnology [to F.W.], King Abdullah University of Science and Technology Grant [KUK-11-008-23 to L.F.], Investissements d'Avenir French Government program French National Research Agency A*MIDEX project [ANR-11-IDEX-0001-02 to M.M.], grant ANR Blanc 1521 01, project RADORDER, the ARC Foundation and OPTITEC to M.M., "Ligue contre le Cancer" [to E.B.G.], and American Cancer Society Research Scholar Grant [RSG-12-161-01-DMC to P.J.B.].

\section{REFERENCES}

(1) Chaurasiya, K. R.; Paramanathan, T.; McCauley, M. J.; Williams, M. C. Biophysical Characterization of DNA Binding From Single Molecule Force Measurements. Physics of Life Reviews 2010, 7, 299341.

(2) De Vlaminck, I.; Dekker, C. Recent Advances in Magnetic Tweezers. Annu. Rev. Biophys. 2012, 41, 453-472.

(3) van Mameren, J.; Peterman, E. J. G.; Wuite, G. J. L. See Me, Feel Me: Methods to Concurrently Visualize and Manipulate Single DNA Molecules and Associated Proteins. Nucleic Acids Res. 2008, 36, 43814389 .

(4) Persson, F.; Tegenfeldt, J. O. DNA in Nanochannels-Directly Visualizing Genomic Information. Chem. Soc. Rev. 2010, 39, 985-999.

(5) Levy, S. L.; Craighead, H. G. DNA Manipulation, Sorting, and Mapping in Nanofluidic Systems. Chem. Soc. Rev. 2010, 39, 11331152.

(6) Reisner, W.; Pedersen, J. N.; Austin, R. H. DNA Confinement in Nanochannels: Physics and Biological Applications. Rep. Prog. Phys. 2012, 75, 106601.

(7) Lam, E. T.; Hastie, A.; Lin, C.; Ehrlich, D.; Das, S. K.; Austin, M. D.; Deshpande, P.; Cao, H.; Nagarajan, N.; Xiao, M.; et al. Genome
Mapping on Nanochannel Arrays for Structural Variation Analysis and Sequence Assembly. Nat. Biotechnol. 2012, 30, 771-776.

(8) Nilsson, A. N.; Emilsson, G.; Nyberg, L. K.; Noble, C.; Stadler, L. S.; Fritzsche, J.; Moore, E. R. B.; Tegenfeldt, J. O.; Ambjornsson, T.; Westerlund, F. Competitive Binding-Based Optical DNA Mapping for Fast Identification of Bacteria - Multi-Ligand Transfer Matrix Theory and Experimental Applications on Escherichia Coli. Nucleic Acids Res. 2014, 42, e118.

(9) Riehn, R.; Lu, M.; Wang, Y. M.; Lim, S. F.; Cox, E. C.; Austin, R. H. Restriction Mapping in Nanofluidic Devices. Proc. Natl. Acad. Sci. U. S. A. 2005, 102, 10012-10016.

(10) Wang, Y. M.; Tegenfeldt, J. O.; Reisner, W.; Riehn, R.; Guan, X. J.; Guo, L.; Golding, I.; Cox, E. C.; Sturm, J.; Austin, R. H. SingleMolecule Studies of Repressor-DNA Interactions Show Long-Range Interactions. Proc. Natl. Acad. Sci. U. S. A. 2005, 102, 9796-9801.

(11) Streng, D. E.; Lim, S. F.; Pan, J.; Karpusenka, A.; Riehn, R. Stretching Chromatin Through Confinement. Lab Chip 2009, 9, 2772-2774.

(12) Zhang, C.; Jiang, K.; Liu, F.; Doyle, P. S.; van Kan, J. A.; van der Maarel, J. R. C. A Nanofluidic Device for Single Molecule Studies with in Situ Control of Environmental Solution Conditions. Lab Chip 2013, 13, 2821-2826.

(13) Zhang, C.; Guttula, D.; Liu, F.; Malar, P. P.; Ng, S. Y.; Dai, L.; Doyle, P. S.; van Kan, J. A.; van der Maarel, J. R. C. Effect of H-NS on the Elongation and Compaction of Single DNA Molecules in a Nanospace. Soft Matter 2013, 9, 9593-9601.

(14) Jiang, K.; Zhang, C.; Guttula, D.; Liu, F.; Van Kan, J. A.; Lavelle, C.; Kubiak, K.; Malabirade, A.; Lapp, A.; Arluison, V.; et al. Effects of Hfq on the Conformation and Compaction of DNA. Nucleic Acids Res. 2015, 43, 4332-4341.

(15) Frykholm, K.; Alizadehheidari, M.; Fritzsche, J.; Wigenius, J.; Modesti, M.; Persson, F.; Westerlund, F. Probing Physical Properties of a DNA-Protein Complex Using Nanofluidic Channels. Small 2014, 10, 884-887.

(16) Frykholm, K.; Berntsson, R. P.-A.; Claesson, M.; de Battice, L.; Odegrip, R.; Stenmark, P.; Westerlund, F. DNA Compaction by the Bacteriophage Protein Cox Studied on the Single DNA Molecule Level Using Nanofluidic Channels. Nucleic Acids Res. DOI: 10.1093/ nar/gkw352. Published Online: April 29, 2016.

(17) Zaitseva, E. M.; Zaitsev, E. N.; Kowalczykowski, S. C. The DNA Binding Properties of Saccharomyces Cerevisiae Rad51 Protein. J. Biol. Chem. 1999, 274, 2907-2915.

(18) Persson, F.; Fritzsche, J.; Mir, K. U.; Modesti, M.; Westerlund, F.; Tegenfeldt, J. O. Lipid-Based Passivation in Nanofluidics. Nano Lett. 2012, 12, 2260-2265.

(19) Odijk, T. The Statistics and Dynamics of Confined or Entangled Stiff Polymers. Macromolecules 1983, 16, 1340-1344.

(20) Odijk, T. Scaling Theory of DNA Confined in Nanochannels and Nanoslits. Phys. Rev. E 2008, 77, 060901.

(21) Dai, L.; van der Maarel, J.; Doyle, P. S. Extended De Gennes Regime of DNA Confined in a Nanochannel. Macromolecules 2014, 47, 2445-2450.

(22) Werner, E.; Mehlig, B. Confined Polymers in the Extended De Gennes Regime. Phys. Rev. E 2014, 90, 062602.

(23) Howard-Flanders, P.; West, S. C.; Stasiak, A. Role of RecA Protein Spiral Filaments in Genetic Recombination. Nature 1984, 309, 215-220.

(24) Prentiss, M.; Prévost, C.; Danilowicz, C. Structure/Function Relationships in RecA Protein-Mediated Homology Recognition and Strand Exchange. Crit. Rev. Biochem. Mol. Biol. 2015, 50, 453-476.

(25) Egelman, E. H. A Robust Algorithm for the Reconstruction of Helical Filaments Using Single-Particle Methods. Ultramicroscopy 2000, 85, 225-234.

(26) Yu, X.; Jacobs, S. A.; West, S. C.; Ogawa, T. Domain Structure and Dynamics in the Helical Filaments Formed by RecA and Rad51 on DNA. Proc. Natl. Acad. Sci. U. S. A. 2001, 98, 8419-8424.

(27) Sheridan, S. D.; Yu, X.; Roth, R.; Heuser, J. E.; Sehorn, M. G.; Sung, P.; Egelman, E. H.; Bishop, D. K. A Comparative Analysis of 
Dmc1 and Rad51 Nucleoprotein Filaments. Nucleic Acids Res. 2008, 36, 4057-4066.

(28) Egelman, E. H.; Stasiak, A. Electron Microscopy of RecA-DNA Complexes: Two Different States, Their Functional Significance and Relation to the Solved Crystal Structure. Micron 1993, 24, 309-324.

(29) Robertson, R. B.; Moses, D. N.; Kwon, Y.; Chan, P.; Chi, P.; Klein, H.; Sung, P.; Greene, E. C. Structural Transitions Within Human Rad51 Nucleoprotein Filaments. Proc. Natl. Acad. Sci. U. S. A. 2009, 106, 12688-12693.

(30) Arenson, T. A.; Tsodikov, O. V.; Cox, M. M. Quantitative Analysis of the Kinetics of End-Dependent Disassembly of RecA Filaments From ssDNA. J. Mol. Biol. 1999, 288, 391-401.

(31) Modesti, M.; Ristic, D.; van der Heijden, T.; Dekker, C.; van Mameren, J.; Peterman, E. J. G.; Wuite, G. J. L.; Kanaar, R.; Wyman, C. Fluorescent Human RAD51 Reveals Multiple Nucleation Sites and Filament Segments Tightly Associated Along a Single DNA Molecule. Structure 2007, 15, 599-609.

(32) Hilario, J.; Amitani, I.; Baskin, R. J.; Kowalczykowski, S. C. Direct Imaging of Human Rad51 Nucleoprotein Dynamics on Individual DNA Molecules. Proc. Natl. Acad. Sci. U. S. A. 2009, 106, 361-368.

(33) van Mameren, J.; Modesti, M.; Kanaar, R.; Wyman, C.; Peterman, E. J. G.; Wuite, G. J. L. Counting RAD51 Proteins Disassembling From Nucleoprotein Filaments Under Tension. Nature 2009, 457, 745-748.

(34) Bugreev, D. V.; Mazin, A. V. Ca2+ Activates Human Homologous Recombination Protein Rad51 by Modulating Its ATPase Activity. Proc. Natl. Acad. Sci. U. S. A. 2004, 101, 9988-9993.

(35) Fornander, L. H.; Frykholm, K.; Reymer, A.; Renodon-Corniere, A.; Takahashi, M.; Nordén, B. Ca2+ Improves Organization of SingleStranded DNA Bases in Human Rad51 Filament, Explaining Stimulatory Effect on Gene Recombination. Nucleic Acids Res. 2012, 40, 4904-4913.

(36) Atwell, S.; Disseau, L.; Stasiak, A. Z.; Stasiak, A.; RenodonCorniere, A.; Takahashi, M.; Viovy, J. L.; Cappello, G. Probing Rad51DNA Interactions by Changing DNA Twist. Nucleic Acids Res. 2012, 40, 11769-11776.

(37) Miné, J.; Disseau, L.; Takahashi, M.; Cappello, G.; Dutreix, M.; Viovy, J.-L. Real-Time Measurements of the Nucleation, Growth and Dissociation of Single Rad51-DNA Nucleoprotein Filaments. Nucleic Acids Res. 2007, 35, 7171-7187.

(38) Candelli, A.; Holthausen, J. T.; Depken, M.; Brouwer, I.; Franker, M. A. M.; Marchetti, M.; Heller, I.; Bernard, S.; Garcin, E. B.; Modesti, M.; et al. Visualization and Quantification of Nascent RAD51 Filament Formation at Single-Monomer Resolution. Proc. Natl. Acad. Sci. U. S. A. 2014, 111, 15090-15095.

(39) Galletto, R.; Amitani, I.; Baskin, R. J.; Kowalczykowski, S. C. Direct Observation of Individual RecA Filaments Assembling on Single DNA Molecules. Nature 2006, 443, 875-878.

(40) van der Heijden, T.; Seidel, R.; Modesti, M.; Kanaar, R.; Wyman, C.; Dekker, C. Real-Time Assembly and Disassembly of Human RAD51 Filaments on Individual DNA Molecules. Nucleic Acids Res. 2007, 35, 5646-5657.

(41) van der Heijden, T.; Van Noort, J.; van Leest, H.; Kanaar, R.; Wyman, C.; Dekker, N. H.; Dekker, C. Torque-Limited RecA Polymerization on dsDNA. Nucleic Acids Res. 2005, 33, 2099-2105.

(42) Tombline, G. Biochemical Characterization of the Human RAD51 Protein. II. Adenosine Nucleotide Binding and Competition. J. Biol. Chem. 2002, 277, 14426-14433.

(43) Arata, H.; Dupont, A.; Mine-Hattab, J.; Disseau, L.; RenodonCorniere, A.; Takahashi, M.; Viovy, J. L.; Cappello, G. Direct Observation of Twisting Steps During Rad51 Polymerization on DNA. Proc. Natl. Acad. Sci. U. S. A. 2009, 106, 19239-19244.

(44) Gibb, B.; Silverstein, T. D.; Finkelstein, I. J.; Greene, E. C. Single-Stranded DNA Curtains for Real-Time Single-Molecule Visualization of Protein-Nucleic Acid Interactions. Anal. Chem. 2012, 84, $7607-7612$.
(45) van der Walt, S.; Schönberger, J. L.; Nunez-Iglesias, J.; Boulogne, F.; Warner, J. D.; Yager, N.; Gouillart, E.; Yu, T. ScikitImage: Image Processing in Python. PeerJ 2014, 2, e453.

(46) Chambolle, A. An Algorithm for Total Variation Minimization and Applications. J. Math. Imaging Vision 2004, 20, 89-97.

(47) Persson, F.; Utko, P.; Reisner, W.; Larsen, N. B.; Kristensen, A. Confinement Spectroscopy: Probing Single DNA Molecules with Tapered Nanochannels. Nano Lett. 2009, 9, 1382-1385.

(48) Lee, J. W.; Cox, M. M. Inhibition of RecA Protein Promoted ATP Hydrolysis. 2. Longitudinal Assembly and Disassembly of RecA Protein Filaments Mediated by ATP and ADP. Biochemistry 1990, 29, $7677-7683$

(49) Prasad, T. K.; Yeykal, C. C.; Greene, E. C. Visualizing the Assembly of Human Rad51 Filaments on Double-Stranded DNA. J. Mol. Biol. 2006, 363, 713-728.

(50) Burkhardt, T. W.; Yang, Y.; Gompper, G. Fluctuations of a Long, Semiflexible Polymer in a Narrow Channel. Phys. Rev. E 2010, $82,041801$.

(51) Murphy, M. C.; Rasnik, I.; Cheng, W.; Lohman, T. M.; HA, T. Probing Single-Stranded DNA Conformational Flexibility Using Fluorescence Spectroscopy. Biophys. J. 2004, 86, 2530-2537.

(52) Ristic, D.; Modesti, M.; van der Heijden, T.; van Noort, J.; Dekker, C.; Kanaar, R.; Wyman, C. Human Rad51 Filaments on Double-and Single-Stranded DNA: Correlating Regular and Irregular Forms with Recombination Function. Nucleic Acids Res. 2005, 33, 3292-3302.

(53) Baumann, P.; Benson, F. E.; West, S. C. Human Rad51 Protein Promotes ATP-Dependent Homologous Pairing and Strand Transfer Reactions in Vitro. Cell 1996, 87, 757-766.

(54) Sung, P.; Krejci, L.; Van Komen, S.; Sehorn, M. G. Rad51 Recombinase and Recombination Mediators. J. Biol. Chem. 2003, 278, 42729-42732.

(55) Sung, P.; Klein, H. Mechanism of Homologous Recombination: Mediators and Helicases Take on Regulatory Functions. Nat. Rev. Mol. Cell Biol. 2006, 7, 739-750. 